\title{
Amorphous Calcium Carbonate
}

National Cancer Institute

\section{Source}

National Cancer Institute. Amorphous Calcium Carbonate. NCI Thesaurus. Code C148184.

A nutritional supplement composed of a proprietary synthetic and amorphous form of calcium carbonate, that can potentially be used to replenish calcium. Upon administration of amorphous calcium carbonate (ACC), the mineral calcium may give calcium supplementary support, improve bone, muscle and heart strength, improve performance and decrease bone-associated pain. 